\title{
Graphs and (Levels of) Cooperation in Games: Two Ways How to Allocate the Surplus
}

\author{
O. Tejada ${ }^{1}$ and M. Álvarez-Mozos*2 \\ ${ }^{1}$ CER-ETH Center of Economic Research, ETH Zurich, Switzerland. \\ ${ }^{2}$ Department de Matemàtica Econòmica Financera i Actuarial, Universitat de Barcelona and \\ BEAT, Spain.
}

Published in Mathematical Social Sciences (2018)

Published version available at http://www.sciencedirect.com/

DOI: $10.1016 /$ j.mathsocsci.2018.02.005

\begin{abstract}
We analyze surplus allocation problems where cooperation between agents is restricted both by a communication graph and by a sequence of embedded partitions of the agent set. For this type of problem, we define and characterize two new values extending the Shapley value and the Banzhaf value, respectively. Our results enable the axiomatic comparison between the two values and provide some basic insights for the analysis of fair resource allocation in today's fully integrated societies.
\end{abstract}

Keywords: Coalitional games; Graph-restricted communication; Levels structure; Shapley value; Banzhaf value

${ }^{*}$ Corresponding author. E-mail address: mikel.alvarez@ub.edu 


\section{Introduction}

As a general rule, the layout of political administrations consist of a series of embedded layers. The EU, for instance, is integrated by countries, which are organized in regions, which, in turn, are divided into smaller administrative units, say cities, and so on. As far as his/her relation with political institutions - and hence with political power - is concerned, a EU citizen can typically deal with one institution only at each level. The overall political and administrative layout typically affects the possibilities of citizens to cooperate: there would be no administrative hurdles if no political institution existed. For example, it is generally easier to engage in a business venture within a common labor market under a single set of rules than across several markets, each regulated by a different set of rules. ${ }^{1}$

The fact that citizens are integrated in a sequence of embedded layers does not prevent them from interacting in various other ways, some of which go beyond the lines set up by political institutions. For instance, there is a great flow of workers between the major financial markets even though they do not belong to a common political entity. This is possible due to the existence of certain networks, very especially transportation and communication networks. More generally, trade between individuals and firms does not necessarily submit to political structures, especially after the outset of globalization. Rivers, highways, train networks, among others, facilitate business and enable individuals and firms to use their full potential. The production chains of some major corporations, in particular, are now distributed over several countries, thanks to the existence of many global networks. With such non-political linkages boosting economic growth, most of the redistribution policies - say, taxes and public expenditures - are, however, decided only by political institutions operating at national or regional level.

In a framework where production is not only intertwined with political institutions but also with a host of other (binary) linkages such as social networks, is there a

\footnotetext{
${ }^{1}$ The so-called border effect is a well-documented phenomenon in international trade (see e.g. Evans, 2003).
} 
reasonable way to address the issue of how the aggregate spoils generated by all citizens should be shared? Providing knowledge about solutions to this problem is the general object of this paper. Taking here mainly a normative approach, we focus on certain properties that may be required for surplus allocation rules. In this vein, it is worth noting that the increase in inequality (see e.g. Atkinson, 2015) that has taken place in the past few years in many countries has raised a major concern: policy decisions should be adopted to guarantee that all citizens benefit from globalization. Our analysis features some elements of fairness that revolve around this concern.

To elaborate, we analyze the class of surplus allocation problems in which cooperation between agents - say, citizens - is restricted both by a sequence of embedded partitions of the agent set and by a communication graph between agents. Formally, we consider a triple made up of a cooperative game, a levels structure, and a nondirected graph, which we call a Game with Graph-Restricted Communication and Levels Structure of Cooperation. First, the cooperative game describes the potential gains that any subset of players can attain on their own, assuming that cooperation is unrestricted. Second, the sequence of embedded partitions of the player set (the so-called levels structure) represents the different "administrative units" in which players are organized. These given arrangements among agents restrict or hinder the formation of coalitions where some of its members belong to different units at some levels. Third and last, the communication graph accounts for the bilateral relations that may exist between players, e.g. due to commercial relations. We assume that a coalition of agents can cooperate only if all of its members are path-connected within the graph. Such links extend naturally to higher units: A city is connected with another city if there is a link between their citizens. Likewise the levels structure, a communication graph affects cooperation between all players, whether directly or indirectly.

Games with graph-restricted communication and levels structure of cooperation provide an appropriate model to address the normative problem of how to allocate the surplus that all the agents can potentially create under the restrictions placed by all layers of political institutions and all communication networks. To make progress in the analysis of this problem, we introduce two values (or point-valued solutions) for 
games with graph-restricted communication and levels structure of cooperation. The first value focuses on the orderings in which coalitions are formed, while the second value considers coalitions directly, without any reference to how they are formed. As is standard in the literature, the first value extends the Shapley value and the second value extends the Banzhaf value, the two classic solutions for cooperative games. We then provide a characterization of each value by means of several properties (or axioms). Such properties are of two types. A first type describes particular ways how the surplus sharing should be affected by alterations of the communication graph. A second set of properties deals with changes in the levels structure. Importantly, the latter properties used in either characterization result are (logically) comparable. This fact may be useful when asking whether to use one value or the other for a particular situation. ${ }^{2}$

Our contribution belongs to the extensive literature of games with restricted cooperation, which dates back at least to Aumann and Drèze (1974) (see also Owen, 1977; Myerson, 1977). Owen (1977) considers situations in which cooperation is restricted by a partition of the player set-the so-called games with coalition structure-, which are particular instances of the more general games with coalition configuration (see Albizuri et al., 2006; Albizuri and Aurrekoetxea, 2006; Andjiga and Courtin, 2015). In the latter model, coalitions are not necessarily disjoint. In turn, Myerson (1977) considers situations in which cooperation is restricted by a communication graph, the so-called games with graph-restricted communication. Several further papers have built on these models or extended them (see e.g. Owen, 1986; Amer et al., 2002; Alonso-Meijide and FiestrasJaneiro, 2002, 2006). Singularly, Winter (1989) generalized the model of games with coalition structure to include restrictions to cooperation that may exist at various levels. He refers to his extended framework as games with levels structure (of cooperation). Both types of restrictions to cooperation, i.e. levels structures and undirected graphs, can, however, exist simultaneously. To account for this possibility, Vázquez-Brage et al. (1996) and Alonso-Meijide et al. (2009) have already proposed and characterized generalizations of the Shapley value and the Banzhaf value for games with both a coalition structure and a communication graph. The model we analyze is a natural generaliza-

\footnotetext{
${ }^{2}$ We elaborate on this issue in Section 3.3.
} 
tion of the latter, insofar as it considers a levels structure instead of coalition structure (i.e, a levels structure with a single level).

The paper is organized as follows: In Section 2 we set the notation and introduce the main concepts from the literature. In Section 3 we define two new values for games with graph-restricted communication and levels structure of cooperation, which we characterize by a number of properties and then compare. Section 4 concludes. The proofs are given in the Appendix.

\section{Notations and Preliminaries}

\subsection{Cooperative games with transferable utility}

Let $\Omega$ denote the (possibly infinite) set of potential players. A cooperative game with transferable utility (or simply a game) is a pair $(N, v)$, where $\emptyset \neq N \subseteq \Omega$ is a finite set of players and $v: 2^{N}=\{S: S \subseteq N\} \rightarrow \mathbb{R}$ is the characteristic function, with $v(\emptyset)=0$. For every coalition $S \subseteq N, v(S)$ represents the worth of coalition $S$, i.e., the total payoff that members of the coalition can obtain by agreeing to cooperate. We denote the collection of all games by $\mathcal{G}$. For the sake of readability, we henceforth abuse notation slightly and write $T \cup i$ and $T \backslash i$ instead of $T \cup\{i\}$ and $T \backslash\{i\}$ for $T \subseteq N$ and $i \in N$, respectively. We use the $|\cdot|$ operator to denote the cardinality of a finite set.

A value on $\mathcal{G}$ is a map, $\mathrm{f}$, that assigns a unique vector $\mathrm{f}(N, v) \in \mathbb{R}^{N}$ to every $(N, v) \in \mathcal{G}$. A permutation of $N$ is a bijective map $\pi: N \rightarrow N$. Let $\Pi(N)$ denote the set of permutations of $N$. Given $\pi \in \Pi(N)$ and $i \in N$, let $\pi^{-1}[i]$ indicate the set of players ordered before $i$ in permutation $\pi$, i.e., $\pi^{-1}[i]=\{j \in N: \pi(j)<\pi(i)\}$. Next, we present the formal definitions of two well-known values on $\mathcal{G}$, namely the Shapley and Banzhaf values.

Definition 2.1. The Shapley value (Shapley, 1953), Sh, is the value on $\mathcal{G}$ defined for $\operatorname{every}(N, v) \in \mathcal{G}$ and $i \in N$ by

$$
\mathrm{Sh}_{i}(N, v)=\frac{1}{|\Pi(N)|} \sum_{\pi \in \Pi(N)}\left[v\left(\pi^{-1}[i] \cup i\right)-v\left(\pi^{-1}[i]\right)\right] .
$$


The Banzhaf value (Banzhaf, 1965), $\mathrm{Ba}$, is the value on $\mathcal{G}$ defined for every $(N, v) \in \mathcal{G}$ and $i \in N$ by

$$
\mathrm{Ba}_{i}(N, v)=\frac{1}{2^{|N|-1}} \sum_{S \subseteq N \backslash i}[v(S \cup i)-v(S)] .
$$

The differences between these values are well know from an axiomatic viewpoint (see e.g. Young, 1985; Feltkamp, 1995; Nowak, 1997). We note that the Shapley value can be alternatively defined as

$$
\mathrm{Sh}_{i}(N, v)=\sum_{S \subseteq N \backslash i} \frac{|S| !|N \backslash(S \cup i)| !}{|N| !}[v(S \cup i)-v(S)] .
$$

\subsection{Games with graph-restricted communication}

A communication graph is an undirected graph without loops defined on a finite set of nodes. That is, $(N, C)$ is a communication graph if $N$ is a finite set of nodes and $C$ is a set of links among the nodes. A link between $i$ and $j$ is denoted by $\{i: j\}$ (note that $\{i: j\}=\{j: i\})$. Given $i, j \in S \subseteq N$, we say that $i$ and $j$ are path-connected (or just connected) in $S$ by $C$ if there is a path in $S$ connecting them, i.e., if for some $k \geq 0$, there is a subset $\left\{i_{0}, \ldots, i_{k}\right\} \subseteq S$ such that $i_{0}=i, i_{k}=j$, and for every $l \in\{1, \ldots k\},\left\{i_{l-1}: i_{l}\right\} \in C$. By $S / C$ we denote the partition of $S$ into maximal connected components. The complete graph on a finite set $N$ is denoted by $\left(N, C^{N}\right)$, i.e., $C^{N}=\{\{i: j\}: i, j \in N, i \neq j\}$. The set of all communication graphs is denoted by $\mathcal{C}$. Given a communication graph $(N, C) \in \mathcal{C}$, a node $i \in N$ is said to be isolated in the graph if there is no link from her, i.e., if for every $j \in N \backslash i,\{i: j\} \notin C$. The communication graph $\left(N, C^{-i}\right)$ is obtained from $(N, C)$ by dissolving the links in which $i$ is involved, i.e., $C^{-i}=\{\{k: l\} \in C: k, l \in N \backslash i\}$. Similarly, given a link $\{i: j\}$, the communication graph $\left(N, C^{-i j}\right)$ is obtained from $(N, C)$ by eliminating the link $\{i: j\}$, i.e., $C^{-i j}=\{\{k: l\} \in C:\{k: l\} \neq\{i: j\}\}$.

A game with graph-restricted communication is a triple $(N, v, C)$ where $(N, v) \in \mathcal{G}$ and $(N, C) \in \mathcal{C}$. The set of all games with graph-restricted communication is denoted by $\mathcal{G C}$. A value on $\mathcal{G C}$ is a map, $\mathrm{f}$, that assigns a unique vector $\mathrm{f}(N, v) \in \mathbb{R}^{N}$ to every $(N, v, C) \in \mathcal{G C}$. 
For every $(N, v, C) \in \mathcal{G C}$, the graph-restricted game $\left(N, v^{C}\right) \in \mathcal{G}$ assumes that players can only communicate through $(N, C)$. In other words, a coalition can cooperate only if it is connected through the communication graph. Formally, for every $S \subseteq N$,

$$
v^{C}(S)=\sum_{T \in S / C} v(T) .
$$

Two well-known values on $\mathcal{G C}$ that generalize the Shapley and Banzhaf values are presented below.

Definition 2.2. The Myerson value (Myerson, 1977), SG, is the value on $\mathcal{G C}$ defined for every $(N, v, C) \in \mathcal{G C}$ by

$$
\mathrm{SG}(N, v, C)=\operatorname{Sh}\left(N, v^{C}\right) .
$$

The Banzhaf graph value (Owen, 1986), BG, is the value on $\mathcal{G C}$ defined for every $(N, v, C) \in \mathcal{G C}$ by

$$
\mathrm{BG}(N, v, C)=\mathrm{Ba}\left(N, v^{C}\right) .
$$

\subsection{Games with levels structure of cooperation}

Consider now that the cooperation among agents is restricted by means of a finite sequence of partitions defined on the player set, each of them being coarser than the previous one. Formally, Winter (1989) introduced a levels structure of cooperation (or simply a levels structure) being a pair $(N, \underline{B})$, where $N \subseteq \Omega$ is a finite set of players and $\underline{B}$ is a sequence of partitions of $N, \underline{B}=\left\{B_{0}, \ldots, B_{k+1}\right\}$, with the following properties: $B_{0}=\{\{i\}: i \in N\}, B_{k+1}=\{N\}$, and, for each $r \in\{0, \ldots, k\}, B_{r+1}$ is coarser than $B_{r}$. That is to say, for each $r \in\{1, \ldots, k+1\}$ and each $S \in B_{r}$, there is $B \subseteq B_{r-1}$ such that $S=\cup_{U \in B} U$. Each $S \in B_{r}$ is called a union and $B_{r}$ is called the $r^{\text {th }}$ level of $\underline{B}$. The levels $B_{0}$ and $B_{k+1}$ are added for notational convenience. We denote by $\left(N, \underline{B_{0}}\right)$ the trivial levels structure with $k=0$, i.e., $\underline{B_{0}}=\left\{B_{0}, B_{1}\right\}$, where $B_{0}=\{\{i\}\}_{i \in N}$ and $B_{1}=\{N\}$. We further denote by $\mathcal{L}$ the set of all levels structures. Let $(N, \underline{B}) \in \mathcal{L}$ with $\underline{B}=\left\{B_{0}, \ldots, B_{k+1}\right\}$ and $i \in N$. Then, $\left(N, \underline{B^{-i}}\right) \in \mathcal{L}(N)$ is the levels structure obtained from $(N, \underline{B})$ by isolating player $i$ from the union she belongs 
to at each level, i.e., $\underline{B^{-i}}=\left\{B_{0}, B_{1}^{-i}, \ldots, B_{k}^{-i}, B_{k+1}\right\}$, where, for all $r \in\{1, \ldots, k\}$, $B_{r}^{-i}=\left\{U \in B_{r}: i \notin U\right\} \cup\{S \backslash i,\{i\}\}$ with $i \in S \in B_{r}$.

A game with levels structure of cooperation is a triple $(N, v, \underline{B})$, where $(N, v) \in \mathcal{G}$ and $(N, \underline{B}) \in \mathcal{L}$. We denote by $\mathcal{G} \mathcal{L}$ the set of all games with levels structure of cooperation. Given $(N, v, \underline{B}) \in \mathcal{G L}$ with $\underline{B}=\left\{B_{0}, \ldots, B_{k+1}\right\}$, for each $r \in\{0, \ldots, k\}$ we define the $r^{\text {th }}$-level union game $\left(B_{r}, v^{r}, \underline{B^{r}}\right) \in \mathcal{G} \mathcal{L}$ induced from $(N, v, \underline{B})$ as the game with levels structure of cooperation with the elements of $B_{r}$ as players, characteristic function $v^{r}$ given by $v^{r}(S)=v\left(\bigcup_{U \in S} U\right)$ for any coalition $S \subseteq B_{r}$, and with levels structure $\underline{B^{r}}=\left\{B_{0}^{r}, \ldots, B_{k-r+1}^{r}\right\}$ given by $B_{0}^{r}=\left\{\{U\}: U \in B_{r}\right\}, B_{s}^{r}=\left\{\left\{U \in B_{r}: U \subseteq U^{\prime}\right\}:\right.$ $\left.U^{\prime} \in B_{r+s}\right\}$ for $s \in\{1, \ldots, k-r\}$, and $B_{k-r+1}^{r}=\left\{\left\{U: U \in B_{k}\right\}\right\}$. Note that $\underline{B^{r}}=\underline{B}$ if $r=0$, whereas $\underline{B^{k}}$ is the trivial levels structure $\underline{B_{0}}$ on the player set $\left\{U: U \in B_{k}\right\}$.

A value on $\mathcal{G} \mathcal{L}$ is a map, $\mathrm{f}$, that assigns to every game with levels structure of cooperation $(N, v, \underline{B}) \in \mathcal{G} \mathcal{L}$ a vector $\mathrm{f}(N, v, \underline{B}) \in \mathbb{R}^{N}$. To present two values on $\mathcal{G} \mathcal{L}$, we need to introduce some further notation. On the one hand, for every $(N, \underline{B}) \in \mathcal{L}$ with $\underline{B}=\left\{B_{0}, \ldots, B_{k+1}\right\}$, the set of permutations of $N$ that respect the levels structure $(N, \underline{B})$ is denoted by $\Omega(\underline{B})$ and is defined by

$$
\begin{aligned}
\Omega(\underline{B})= & \left\{\pi \in \Pi(N): \forall B_{r} \in \underline{B}, \forall T \in B_{r}, \forall i, j \in T,\right. \\
& \text { and } k \in N, \text { if } \pi(i)<\pi(k)<\pi(j) \text { then } k \in T\} .
\end{aligned}
$$

On the other hand, for every $(N, \underline{B}) \in \mathcal{L}$ and $i \in N$, let $i \in U_{0}=\{i\} \subseteq U_{1} \subseteq \cdots \subseteq$ $U_{k} \subseteq U_{k+1}$ such that $U_{r} \in B_{r}$ for all $r \in\{0, \ldots, k+1\}$. Then, the partition induced by $\underline{B}$ on $i$ is defined as

$$
P(i, \underline{B})=\bigcup_{r=0}^{k}\left(B_{r}\right)_{\mid U_{r+1} \backslash U_{r}} .
$$

Then, $P(i, \underline{B}) \in \mathcal{P}(N \backslash i)$. We denote $|P(i, \underline{B})|$ by $m_{i}$, and the unions of the partition induced by $\underline{B}$ on $i$, by $P(i, \underline{B})=\left\{T_{1}, \ldots, T_{m_{i}}\right\}$. Finally, the set of indices of the partition induced by $\underline{B}$ on $i$ is denoted by $M_{i}=\left\{1, \ldots, m_{i}\right\}$, and for every $R \subseteq M_{i}, T_{R}=\cup_{r \in R} T_{r}$.

Definition 2.3. The Shapley levels value (Winter, 1989), $\mathrm{Sh}^{\mathrm{L}}$, is the value on $\mathcal{G} \mathcal{L}$ 
defined for every $(N, v, \underline{B}) \in \mathcal{G C}$ and $i \in N$ by

$$
\operatorname{Sh}_{i}^{\mathrm{L}}(N, v, \underline{B})=\frac{1}{|\Omega(\underline{B})|} \sum_{\sigma \in \Omega(\underline{B})}\left[v\left(\sigma^{-1}[i] \cup i\right)-v\left(\sigma^{-1}[i]\right)\right] .
$$

The Banzhaf levels value (Álvarez-Mozos and Tejada, 2011), $\mathrm{Ba}^{\mathrm{L}}$, is the value on $\mathcal{G} \mathcal{L}$ defined for every $(N, v, C) \in \mathcal{G C}$ and $i \in N$ by

$$
\mathrm{Ba}_{i}^{\mathrm{L}}(N, v, \underline{B})=\sum_{R \subseteq M_{i}} \frac{1}{2^{m_{i}}}\left[v\left(T_{R} \cup i\right)-v\left(T_{R}\right)\right] .
$$

The two values above yield the Shapley value and the Banzhaf value, respectively, if the levels structure is trivial. They also generalize the Owen value (Owen, 1977) and the Banzhaf-Owen value (Owen, 1982). ${ }^{3}$

\section{Two New Solutions}

We are now in a position to introduce the main mathematical objects we shall deal with. A game with graph-restricted communication and levels structure of cooperation (henceforth, a graph-levels restricted game) is a four-tuple $(N, v, C, \underline{B}) \in \mathcal{G C} \mathcal{L}$ where $N \subseteq \Omega,(N, v) \in \mathcal{G},(N, C) \in \mathcal{C}$ and $(N, \underline{B}) \in \mathcal{L}$. We let $\mathcal{G C} \mathcal{L}$ denote the set of all graph-levels restricted games. A value on $\mathcal{G C L}$ is then a map, $\mathrm{f}$, that assigns to every game with graph-restricted communication and levels structure of cooperation $(N, v, C, \underline{B}) \in \mathcal{G C} \mathcal{L}$ a vector $\mathrm{f}(N, v, C, \underline{B}) \in \mathbb{R}^{N}$.

In the remainder of this section we do three things. First, we introduce and characterize a new value for graph-levels restricted games, which generalizes the Shapley value. Second, we introduce and characterize another new value for graph-levels restricted games, which generalizes the Banzhaf value. Third, we build on the two characterization results to discuss the differences between the two proposed values. An example is also used to further illustrate these differences.

\footnotetext{
${ }^{3}$ These values are defined in the framework of games with coalition structure.
} 


\subsection{The Myerson levels value}

We start by proposing the natural generalization to our setting of the Shapley value, the Myerson value, and the Shapley levels value.

Definition 3.1. The Myerson levels value, $\mathrm{SG}^{\mathrm{L}}$, is the value on $\mathcal{G C} \mathcal{L}$, defined for every $(N, v, C, \underline{B}) \in \mathcal{G C} \mathcal{L}$ by

$$
\mathrm{SG}^{\mathrm{L}}(N, v, C, \underline{B})=\mathrm{Sh}^{\mathrm{L}}\left(N, v^{C}, \underline{B}\right) .
$$

That is, the Myerson levels value of a graph-levels restricted game, say $(N, v, C, \underline{B})$, is the result of a two-stage procedure. First, the (unrestricted) possibilities of cooperation captured by $(N, v)$ are reduced in accordance with the communication graph $(N, C)$. This means that if there exist returns to scale-i.e., if the game is strictly superadditive-, only coalitions whose members are all connected within the graph can obtain their full potential. ${ }^{4}$ The outcome of this restriction procedure is $\left(N, v^{C}\right)$. Second, the Shapley levels value is applied to the resulting game with levels structure

of cooperation $\left(N, v^{C}, \underline{B}\right)$. This implies that cooperation among agents is further restricted, so that only permutations in which all agents are ordered in a way that respects the levels structure $(N, \underline{B})$ are payoff-relevant.

In the following, we introduce a number of properties that a value for graph-levels restricted games may satisfy.

$\mathrm{CE} \mathrm{A}$ value on $\mathcal{G C} \mathcal{L}, \mathrm{f}$, satisfies component efficiency if for every $(N, v, C, \underline{B}) \in \mathcal{G C} \mathcal{L}$ and every $S \in N / C$,

$$
\sum_{i \in S} \mathrm{f}_{i}(N, v, C, \underline{B})=v(S)
$$

FG A value on $\mathcal{G C} \mathcal{L}, \mathrm{f}$, satisfies fairness in the graph if for every $(N, v, C, \underline{B}) \in \mathcal{G C} \mathcal{L}$ and every $i, j \in U \in B_{1} \in \underline{B}$ such that $\{i: j\} \in C$,

$$
\mathrm{f}_{i}(N, v, C, \underline{B})-\mathrm{f}_{i}\left(N, v, C^{-i j}, \underline{B}\right)=\mathrm{f}_{j}(N, v, C, \underline{B})-\mathrm{f}_{j}\left(N, v, C^{-i j}, \underline{B}\right) .
$$

\footnotetext{
${ }^{4} \mathrm{~A}$ game $(N, v)$ is strictly superadditive if $v(S \cup T)>v(S)+v(T)$ for $\emptyset \neq S, T$ such that $S \cap T=\emptyset$.
} 
$\mathrm{BC}$ A value on $\mathcal{G C} \mathcal{L}, \mathrm{f}$, satisfies balanced contributions if for every $(N, v, C, \underline{B}) \in \mathcal{G C} \mathcal{L}$ and every $i, j \in U \in B_{1} \in \underline{B}$,

$$
\mathrm{f}_{i}(N, v, C, \underline{B})-\mathrm{f}_{i}\left(N, v, C, \underline{B^{-j}}\right)=\mathrm{f}_{j}(N, v, C, \underline{B})-\mathrm{f}_{j}\left(N, v, C, \underline{B^{-i}}\right) .
$$

CLG A value on $\mathcal{G C L}, \mathrm{f}$, satisfies the communication level game property if for every $(N, v, C, \underline{B}) \in \mathcal{G C} \mathcal{L}$ and every $U \in B_{r} \in \underline{B}$,

$$
\sum_{i \in U} \mathrm{f}_{i}(N, v, C, \underline{B})=\mathrm{f}_{U}\left(B_{r},\left(v^{C}\right)^{r}, C^{B_{r}}, \underline{B_{r}}\right) .
$$

The four properties above are inspired by similar properties from the literature. CE and FG were introduced by Myerson (1977) in the framework of games with graphrestricted communication. BC was proposed by Vázquez-Brage et al. (1996) and CLG by Alonso-Meijide et al. (2009) for games with graph-restricted communication and a coalition structure in both cases.

The first property, CE, requires the spoils generated by unrestricted cooperation to be always attainable for coalitions that are maximally connected in the communication graph, regardless of the restrictions imposed by the levels structure. The latter may affect the payoff to specific players but not the total payoff of a maximally connected coalition. Accordingly, there is a sense in which CE builds on the idea that "connected markets" are always efficient. Hence, CE is rather a descriptive property of our setting than a normative requirement.

By contrast, FG is a property with a strong flavor of equity: it requires that the introduction or removal of a communication link between two agents has to affect both players' payoff equally. Redistribution tools such as taxes and public spending could be used to ensure that this condition is fulfilled. Unlike CE, FG does not apply independently of the restrictions imposed by the levels structure, as the two players considered have to belong to the same administrative unit (i.e., union) at all levels. FG is silent with regard to changes in the communication structure that affect citizens who live in different cities, regions or countries.

Like FG, BC applies to any pair of players who belong to the same union at all levels of the levels structure. For such a pair of players, BC requires that when one of the 
players becomes completely isolated from the administrative structure - as modeled by the levels structure - the effect on the other player is always the same (for that union). Hence, for each citizen, BC equalizes the value of the current political structure with respect to the threat that any fellow citizen (who is closest to her in the levels structure) might leave the structure. ${ }^{5}$ In a sense, BC can be seen as a stability property for the lowest non-trivial level of the entire administrative structure: for any two players that belong to the same union, one's threat against the other has to be the same. ${ }^{6}$ Note that $\mathrm{BC}$ assumes that changes in the levels structure induce no changes in the communication graph.

Finally, CLG also deals with the stability of the levels structure, keeping the communication graph fixed. While $\mathrm{BC}$ is concerned with changes in the levels structure from a horizontal perspective (it compares the payoff change of two citizens who belong to the same union at all levels), CLG is concerned with changes in the levels structure from a vertical perspective. Specifically, CLG demands that aggregating units cannot have an effect on the total payoff of the administrative units (i.e., unions) being aggregated.

It turns out that the four properties we introduced single out $\mathrm{SG}^{\mathrm{L}}$.

Theorem 3.1. The Myerson levels value is the unique value on $\mathcal{G C L}$ that satisfies $\mathrm{CE}$, FG, BC, and CLG. Moreover, the four properties are independent. ${ }^{7}$

We point out that this result remains valid if $\mathrm{CE}$ and FG are only demanded when the levels structure is trivial. What is more, we could replace this two properties by any set that characterizes the Myerson value (see, for instance, Myerson, 1980).

\footnotetext{
${ }^{5}$ This interpretation holds generally for the minimal unit in our model, be it composed of citizens or groups of citizens. In the latter case, BC attest to the cohesion of political units.

${ }^{6}$ In combination with CLG, such a notion of stability translates into all other levels of the administrative structure.

${ }^{7}$ For the independence of the properties, we weaken CE and require its applicability only to graphlevels restricted games where the levels structure is trivial.
} 


\subsection{The Banzhaf levels graph value}

Next, we propose one natural generalization to our setting of the Banzhaf value, the Banzhaf graph value, and the Banzhaf levels value.

Definition 3.2. The Banzhaf levels graph value, $\mathrm{BG}^{\mathrm{L}}$, is the value on $\mathcal{G C L}$, defined for every $(N, v, C, \underline{B}) \in \mathcal{G C} \mathcal{L}$ by

$$
\mathrm{BG}^{\mathrm{L}}(N, v, C, \underline{B})=\mathrm{Ba}^{\mathrm{L}}\left(N, v^{C}, \underline{B}\right) .
$$

The Banzhaf levels graph value is obtained by a two-stage procedure similar to the one used to build the Myerson levels value. The main difference is that after the transformation of the players' cooperation possibilities set by the communication graph, which yields a game with levels structure of cooperation, all admissible coalitions are now assumed to be equally likely. This property is characteristic of the Banzhaf value upon which $B G^{\mathrm{L}}$ is based - and is in sharp contrast with the assumption that lies at the foundation of the Shapley value - upon which $\mathrm{SG}^{\mathrm{L}}$ is based - namely that all admissible permutations are equally likely. The differences between the two approaches are further discussed in Section 3.3.

In the following, we introduce more properties that a value for graph-levels restricted games may satisfy.

GI A value on $\mathcal{G C L}, \mathrm{f}$, satisfies graph isolation if for every $(N, v, C, \underline{B}) \in \mathcal{G C L}$ and every $\{i\} \in N / C$,

$$
\mathrm{f}_{i}(N, v, C, \underline{B})=v(\{i\}) .
$$

2-E A value on $\mathcal{G C L}, \mathrm{f}$, satisfies 2-efficiency if for every $(N, v, C, \underline{B}) \in \mathcal{G C} \mathcal{L}$ and every $i, j \in U \in B_{1} \in \underline{B}$ such that $\{i: j\} \in C$,

$$
\mathrm{f}_{i}(N, v, C, \underline{B})+\mathrm{f}_{j}(N, v, C, \underline{B})=\mathrm{f}_{i}\left(N, v_{i j}, C_{i j}, \underline{B}\right)+\mathrm{f}_{j}\left(N, v_{i j}, C_{i j}, \underline{B}\right),
$$

where $C_{i j}=C^{-j} \cup\{\{i: k\}: k \in N \backslash i$, with $\{k: j\} \in C\}$ and for every $S \subseteq N$, $v_{i j}(S)=\left\{\begin{array}{ll}v(S \cup j) & \text { if } i \in S \\ v(S \backslash j) & \text { if } i \notin S\end{array}\right.$. 
NID A value on $\mathcal{G C} \mathcal{L}, \mathrm{f}$, satisfies neutrality under individual desertion if for every $(N, v, C, \underline{B}) \in \mathcal{G C} \mathcal{L}$ and every $i, j \in U \in B_{1} \in \underline{B}$,

$$
\mathrm{f}_{i}(N, v, C, \underline{B})=\mathrm{f}_{i}\left(N, v, C, \underline{B^{-j}}\right) .
$$

1-CLG A value on $\mathcal{G C} \mathcal{L}, \mathrm{f}$, satisfies the 1-communication level game property if for every $(N, v, C, \underline{B}) \in \mathcal{G C} \mathcal{L}$ and every $\{i\} \in B_{r} \in \underline{B}$,

$$
\mathrm{f}_{i}(N, v, C, \underline{B})=\mathrm{f}_{\{i\}}\left(B_{r},\left(v^{C}\right)^{r}, C^{B_{r}}, \underline{B_{r}}\right) .
$$

These four properties are again inspired by similar properties from the literature. GI was introduced by Alonso-Meijide and Fiestras-Janeiro (2006) in the framework of games with graph-restricted communication. 2-E was originally proposed by Lehrer (1988) for games (see also Haller, 1994). The last two properties, NID and 1-CLG, were introduced by Alonso-Meijide et al. (2007) for games with coalition structure.

Because GI is weaker than CE, it allows a similar interpretation. Specifically, GI requires that regardless of any consideration, a player who is isolated in the graph cannot establish cooperation with any other player, and thus her payoff must be equal to her stand-alone worth. Hence, this property builds on the positive assumption that connectedness (in the graph) is a necessary condition for cooperation (in the game). GI also admits a normative interpretation: no player should be allowed to rip off someone else's production if she is isolated in the graph. We stress that GI holds irrespective of the levels structure.

In turn, 2-E deals with the payoff of two players who not only belong together in all levels of the level structure of cooperation but are also directly connected in the graph. Specifically, this property requires that if one of these players delegates her role to another player, their aggregate payoff has to remain the same. Such an internal reorganization may, however, have a re-distributive impact on how much each player is eventually allocated. Note that 2-E assumes both a change in the game and a change in the graph. One rationale for this simultaneous premise is the following: the fact that one player delegates her role to another player means that the former has to be able 
to transfer to the latter a property right on both the production-i.e., on the potential contributions of the game in which the delegating player is involved, provided that the delegated player is also involved - and the network - i.e. on all links in which the delegating player is involved. In particular, if 2-E is satisfied and either transferring the property rights on production - e.g., by signing a contract - or transferring the linkse.g., when they are physical, geographically-based networks - is costly, such internal reorganizations should, in principle, not take place, and thus we should always observe that all players take an active role in the negotiations.

Next, note that NID is stronger than BC. Indeed, while the latter property is a symmetry condition that equalizes the effect that the departure of one player has on any other player who belongs to the same union at all levels, NID requires that these changes be zero. NID thus builds on the normative assumption that the threat of any citizen to unilaterally deviate from the current administrative structure should be void for the fellow citizens who belong to the same unions at all levels.

Finally, 1-CLG is weaker than CLG. Indeed, the former property results from applying the latter property to singleton unions of any level. In particular, the interpretation of 1-CLG is essentially the same as that of CLG.

It turns out that in combination with $\mathrm{FG}$, the four properties above single out $\mathrm{BG}^{\mathrm{L}}$.

Theorem 3.2. The Banzhaf levels graph value is the unique value on $\mathcal{G C} \mathcal{L}$ that satisfies GI, FG, 2-E, NID, and 1-CLG. Moreover, the five properties are independent. ${ }^{8}$

We point out that the result remains valid if GI, 2-E, and FG are only demanded when the levels structure is trivial. What is more, we could replace these two properties by any set that characterizes the Banzhaf graph value.

\subsection{A comparative analysis}

For Theorems 3.1 and 3.2, we have used two types of properties. The first typenamely $\mathrm{CE}$ and $\mathrm{FG}$ for the Myerson levels value $\left(\mathrm{SG}^{\mathrm{L}}\right)$, and $\mathrm{GI}$, FG and 2-E for the

\footnotetext{
${ }^{8}$ For the independence of the properties, we weaken GI and require its applicability only to graphlevels restricted games where the levels structure is trivial.
} 
Banzhaf levels graph value $\left(B G^{\mathrm{L}}\right)$ - is concerned with the issue of how a value should behave with respect to changes in the communication graph. With FG being satisfied by both values, the main difference between the two values stems from which coalitions can attain their full potential: the Myerson levels value assumes that any coalition that is connected in the graph can do so, while the Banzhaf levels graph value imposes conditions relating to efficiency concerns on one- and two-player (connected) coalitions only. From a comparative perspective, the properties of the second type - namely BC and CLG for the Myerson levels value, and NID and 1-CLG for the Banzhaf levels graph value - are also relevant. Indeed, these properties are parallel to each other in the following sense: First, $\mathrm{BC}$ is a weakening of NID and hence $\mathrm{SG}^{\mathrm{L}}$ is less restrictive than $B G^{\mathrm{L}}$ regarding the effect of one agent leaving the levels structure on another. Second, CLG is a stronger requirement than 1-CLG. Accordingly, $S^{L}{ }^{L}$ could be more (less) reasonable than $B G^{\mathrm{L}}$ in those situations where the stability of the levels structure is high (low).

We conclude this section with an example that illustrates both the Myerson levels value and the Banzhaf graph levels value, which also enables a further comparison between both solution concepts.

Example 3.1. Given $N=\{1,2,3,4,5\}$, let $(N, v) \in \mathcal{G}$ be the simple game determined by the set of minimal winning coalitions $M=\{\{1,2,4\},\{1,2,5\},\{3,4,5\}\} .{ }^{9}$ In this game, players 1 and 2 are symmetric and participate in most winning coalitions. In turn, players 4 and 5 are also symmetric, with player 3 being the one who participates in fewer winning coalitions. Then, consider the graph-levels restricted game $(N, v, C, \underline{B}) \in$ $\mathcal{G C} \mathcal{L}$, where the communication graph, $(N, C)$, and the levels structure, $(N, \underline{B})$, are as depicted in Figure 1.

On the one hand, the star shape of the graph describes a situation in which player 1 can enable communication among the remaining players. On the other hand, the levels structure describes a situation in which players 3,4 , and 5 are together in all non-trivial levels of the levels structure, with the other two players joining them sequentially: first

\footnotetext{
${ }^{9}$ That is, for every $S \subseteq N, v(S)=1$ if $T \subseteq S$ for some $T \in M$, and $v(S)=0$ otherwise.
} 


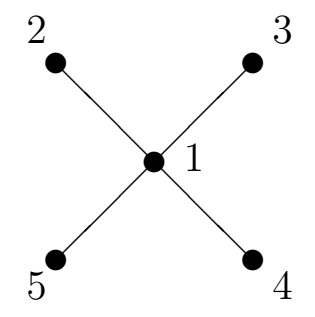

$$
\begin{aligned}
& B_{3}=\{\{1,2,3,4,5\}\}, \\
& B_{2}=\{\{1\},\{2,3,4,5\}\}, \\
& B_{1}=\{\{1\},\{2\},\{3,4,5\}\}, \\
& B_{0}=\{\{1\},\{2\},\{3\},\{4\},\{5\}\} .
\end{aligned}
$$

Figure 1: The communication graph and the levels structure.

player 2, then player 1 .

To compute the Myerson levels value of $(N, v, C, \underline{B})$, we consider the 24 permutations that are admissible given the levels structure, and for each such permutation we identify the player who has a pivotal position with respect to the game with graph-restricted communication. For the computation of the Banzhaf levels graph value, by contrast, we compute the partitions induced by the levels structure, namely $P(1, \underline{B})=\{\{2,3,4,5\}\}$, $P(2, \underline{B})=\{\{1\},\{3,4,5\}\}, P(3, \underline{B})=\{\{1\},\{2\},\{4,5\}\}, P(4, \underline{B})=\{\{1\},\{2\},\{3,5\}\}$, and $P(5, \underline{B})=\{\{1\},\{2\},\{3,4\}\}$. With all these ingredients, we obtain

$$
\begin{aligned}
\operatorname{Sh}^{\mathrm{L}}(N, v, C, \underline{B}) & =\left(\frac{1}{2}, 0, \frac{1}{12}, \frac{5}{24}, \frac{5}{24}\right), \text { and } \\
\operatorname{Ba}^{\mathrm{L}}(N, v, C, \underline{B}) & =\left(\frac{1}{2}, 0, \frac{1}{8}, \frac{1}{4}, \frac{1}{4}\right) .
\end{aligned}
$$

The above outcomes illustrate some of the differences between the players' (relative) payoff as given by the two solution concepts. For instance, according to $\mathrm{Sh}^{\mathrm{L}}$, players 4 and 5 receive more than two times what player 3 is assigned. In contrast, according to $\mathrm{Ba}^{\mathrm{L}}$, the importance of players 4 and 5 is exactly the double of player 3. We also note that the outcome prescribed by the Banzhaf levels graph value is not efficient.

\section{Conclusion}

We have introduced and characterized two new values for graph-levels restricted games. As argued in the Introduction, our analysis offers some worthwhile insights about in- 
come redistribution in today's world economy, in which cooperation between agents is concurrently influenced by embedded political units and networks.

Several aspects of our model deserve scrutiny in the future, of which we mention two. First, a closer mapping of our model to real data may help us discern whether it is the Shapley-like or the Banzhaf-like approach, if any, that is prevalent. Second, a purely non-cooperative model of public finance that implements our two values could guide policy-making, based on the normative considerations raised by our paper.

\section{Acknowledgments}

This research received financial support from Ministerio de Economía y Competitividad through Projects MTM2014-53395-C3-2-P and ECO2014-52340-P as well as from Generalitat de Catalunya through Project 2014-SGR-40. We also want to thank two anonymous reviewers and the Associate Editor for comments that helped to improve the presentation of our results. All remaining errors are our own.

\section{References}

Albizuri, M. J., Aurrecoechea, J., and Zarzuelo, J. M. (2006). Configuration values: Extensions of the coalitional owen value. Games and Economic Behavior, 57:1-17.

Albizuri, M. J. and Aurrekoetxea, J. (2006). Coalition configurations and the banzhaf index. Social Choice and Welfare, 26:571-596.

Alonso-Meijide, J. M., Álvarez-Mozos, M., and Fiestras-Janeiro, M. G. (2009). Values of games with graph restricted communication and a priori unions. Mathematical Social Sciences, 58:202-213.

Alonso-Meijide, J. M., Carreras, F., Fiestras-Janeiro, M. G., and Owen, G. (2007). A comparative axiomatic characterization of the Banzhaf-Owen coalitional value. Decision Support Systems, 43:701-712. 
Alonso-Meijide, J. M. and Fiestras-Janeiro, M. G. (2002). Modification of the Banzhaf value for games with a coalition structure. Annals of Operations Research, 109:213227.

Alonso-Meijide, J. M. and Fiestras-Janeiro, M. G. (2006). The Banzhaf value and communication situations. Naval Research Logistics, 53:198-203.

Álvarez-Mozos, M. and Tejada, O. (2011). Parallel characterizations of a generalized Shapley value and a generalized Banzhaf value for cooperative games with levels structure of cooperation. Decision Support Systems, 52:21-27.

Amer, R., Carreras, F., and Giménez, J. (2002). The modified Banzhaf value for games with coalition structure: an axiomatic caracterization. Mathematical Social Sciences, $43: 45-54$.

Andjiga, N. G. and Courtin, S. (2015). Coalition configurations and share functions. Annals of Operations Research, 225:3-25.

Atkinson, A. B. (2015). Inequality: What Can Be Done? Harvard University Press.

Aumann, R. J. and Drèze, J. (1974). Cooperative games with coalition structures. International Journal of Game Theory, 3:217-237.

Banzhaf, J. F. (1965). Weighted voting does not work: A mathematical analysis. Rutgers Law Review, 19:317-343.

Evans, C. L. (2003). The economic significance of national border effects. The American Economic Review, 93(4):1291-1312.

Feltkamp, V. (1995). Alternative axiomatic characterizations of the Shapley and Banzhaf values. International Journal of Game Theory, 24:179-186.

Haller, H. (1994). Collusion properties of values. International Journal of Game Theory, $23: 261-281$. 
Lehrer, E. (1988). An axiomatization of the Banzhaf value. International Journal of Game Theory, 17:89-99.

Myerson, R. B. (1977). Graphs and cooperation in games. Mathematics of Operations Research, 2:225-229.

Myerson, R. B. (1980). Conference structures and fair allocation rules. International Journal of Game Theory, 9:169-182.

Nowak, A. S. (1997). On the axiomatization of the Banzhaf value without the additivity axiom. International Journal of Game Theory, 26:137-141.

Owen, G. (1977). Values of games with a priori unions. In Henn, R. and Moeschlin, O., editors, Mathematical Economics and Game Theory, pages 76-88. Springer.

Owen, G. (1982). Modification of the Banzhaf-Coleman index for games with a priori unions. In Holler, M., editor, Power, voting and voting power, pages 232-238. Verlag.

Owen, G. (1986). Values of graph-restricted games. SIAM Journal on Algebraic and Discrete Methods, 7:210-220.

Shapley, L. S. (1953). A value for n-person games. In Tucker, A. W., editor, Contributions to the Theory of Games II, pages 307-317. Princeton University Press.

Vázquez-Brage, M., García-Jurado, I., and Carreras, F. (1996). The Owen value applied to games with graph-restricted communication. Games and Economic Behavior, $12: 42-53$.

Winter, E. (1989). A value for cooperative games with levels structure of cooperation. International Journal of Game Theory, 18:227-240.

Young, H. P. (1985). Monotonic Solutions of Cooperative Games. International Journal of Game Theory, 14:65-72. 


\section{Appendix}

\section{Proof of Theorem 3.1.}

The proof proceeds in three steps. First, we prove that the Myerson levels value satisfies CE, FG, BC, and CLG. Second, we prove that there is at most one value on $\mathcal{G C} \mathcal{L}$ satisfying CE, FG, BC, and CLG. Third, we prove that the four properties are logically independent.

\section{Existence}

First, we check that $\mathrm{SG}^{\mathrm{L}}$ satisfies $\mathrm{CE}$. Let $(N, v, C, \underline{B}) \in \mathcal{G C} \mathcal{L}$ and $S \in N / C$. By definition of $v^{C}$, for every $i \in S$ and $T \subseteq N \backslash i$,

$$
v^{C}(T \cup i)-v^{C}(T)=v^{C}((T \cap S) \cup i)-v^{C}(T \cap S)
$$

Then,

$$
\begin{aligned}
& \sum_{i \in S} \mathrm{SG}_{i}^{\mathrm{L}}(N, v, C, \underline{B})=\frac{1}{|\Omega(\underline{B})|} \sum_{\sigma \in \Omega(\underline{B})} \sum_{i \in S}\left[v^{C}\left(\sigma^{-1}[i] \cup i\right)-v^{C}\left(\sigma^{-1}[i]\right)\right] \\
& =\frac{1}{|\Omega(\underline{B})|} \sum_{\sigma \in \Omega(\underline{B})} \sum_{i \in S}\left[v^{C}\left(\left(\sigma^{-1}[i] \cap S\right) \cup i\right)-v^{C}\left(\sigma^{-1}[i] \cap S\right)\right] \\
& =\frac{1}{|\Omega(\underline{B})|} \sum_{\sigma \in \Omega(\underline{B})} v^{C}(S)=v(S),
\end{aligned}
$$

where the first equality is obtained by switching the order of the summations, the second equality results from applying Eq. (1), and the last equality follows by definition of $v^{C}$ and the observation that for every ordering of the agents in $S$, adding the marginal contributions of every player to her set of predecessors yields $v^{C}(S)-v^{C}(\emptyset)=v^{C}(S)$. Indeed, let $S=\left\{i_{1}, \ldots, i_{s}\right\}$, with $s=|S|$, be such that $\sigma^{-1}\left[i_{j}\right]<\sigma^{-1}\left[i_{l}\right]$ if and only if $j<l$. Then

$$
\begin{aligned}
& \sum_{i \in S}\left[v^{C}\left(\left(\sigma^{-1}[i] \cap S\right) \cup i\right)-v^{C}\left(\sigma^{-1}[i] \cap S\right)\right] \\
= & \sum_{l=1}^{s}\left[v^{C}\left(i_{1} \cup \ldots \cup i_{l}\right)-v^{C}\left(i_{1} \cup \ldots \cup i_{l-1}\right)\right]=v^{C}\left(i_{1} \cup \ldots \cup i_{s}\right) .
\end{aligned}
$$


Second, we check that $\mathrm{SG}^{\mathrm{L}}$ satisfies FG. Let $(N, v, C, \underline{B}) \in \mathcal{G C} \mathcal{L}$ and $i, j \in U \in B_{1} \in$ $\underline{B}$ be such that $\{i: j\} \in C$. Define $w=v^{C}-v^{C^{-i j}}$. Since $\mathrm{SG}^{\mathrm{L}}$ is additive (see pp 229-230 in Winter, 1989),

$$
\begin{aligned}
\mathrm{SG}_{i}^{\mathrm{L}}(N, v, C, \underline{B})-\mathrm{SG}_{i}^{\mathrm{L}}\left(N, v, C^{-i j}, \underline{B}\right) & =\mathrm{Sh}_{i}^{\mathrm{L}}\left(N, v^{C}, \underline{B}\right)-\mathrm{Sh}_{i}^{\mathrm{L}}\left(N, v^{C^{-i j}}, \underline{B}\right) \\
& =\mathrm{Sh}_{i}^{\mathrm{L}}(N, w, \underline{B}) .
\end{aligned}
$$

We show that $i$ and $j$ are symmetric players in $(N, w)$. Indeed, let $S \subseteq N \backslash\{i, j\}$. Then, because $(S \cup i) / C=(S \cup i) / C^{-i j}$ and $(S \cup j) / C=(S \cup j) / C^{-i j}$, it must be that

$$
w(S \cup i)=w(S \cup j)=0 .
$$

Next, since $i, j \in U \in B_{1} \in \underline{B}$ and $\mathrm{Sh}^{\mathrm{L}}$ satisfies symmetry (see pp 23-24 in ÁlvarezMozos and Tejada, 2011), $\operatorname{Sh}_{i}^{\mathrm{L}}(N, w, \underline{B})=\operatorname{Sh}_{j}^{\mathrm{L}}(N, w, \underline{B})$. Finally, observe that in Eq. (2) the roles of $i$ and $j$ can be replaced. Combining the last equality with these two versions of Eq. (2), we obtain that $\mathrm{SG}^{\mathrm{L}}$ satisfies FG.

Third, $\mathrm{SG}^{\mathrm{L}}$ satisfies $\mathrm{BC}$ by its definition and the fact that $\mathrm{Sh}^{\mathrm{L}}$ satisfies the level balanced contributions property (see p 24 in Álvarez-Mozos and Tejada, 2011).

Fourth and last, $\mathrm{SG}^{\mathrm{L}}$ satisfies CLG because for every $U \in B_{r} \in \underline{B}$,

$$
\begin{aligned}
\sum_{i \in U} \mathrm{SG}_{i}^{\mathrm{L}}(N, v, C, \underline{B}) & =\sum_{i \in U} \operatorname{Sh}_{i}^{\mathrm{L}}\left(N, v^{C}, \underline{B}\right)=\mathrm{Sh}_{U}^{\mathrm{L}}\left(B_{r},\left(v^{C}\right)^{r}, \underline{B_{r}}\right) \\
& =\operatorname{Sh}_{U}^{\mathrm{L}}\left(B_{r},\left(\left(v^{C}\right)^{r}\right)^{C^{B_{r}}}, \underline{B_{r}}\right)=\mathrm{SG}_{U}^{\mathrm{L}}\left(B_{r},\left(v^{C}\right)^{r}, C^{B_{r}}, \underline{B_{r}}\right),
\end{aligned}
$$

where the first and last equalities are by definition of $\mathrm{SG}^{\mathrm{L}}$, the second one is due to the fact that $\mathrm{Sh}^{\mathrm{L}}$ satisfies the level game property (see p 24 in Álvarez-Mozos and Tejada, 2011), and the third holds from the observation that when the communication graph is complete, the game with graph-restricted communication corresponds to the game itself.

\section{Uniqueness}

Let $\mathrm{f}$ be a value on $\mathcal{G C} \mathcal{L}$ satisfying the four properties and let $(N, v, C, \underline{B}) \in \mathcal{G C} \mathcal{L}$ be a graph-levels restricted game with $k$ levels. We show uniqueness of $\mathrm{f}(N, v, C, \underline{B})$ by induction on $k$. 
First, let $k=0$, i.e., $\underline{B}=\underline{B_{0}}$. Define $\mathrm{fc}$, a value on $\mathcal{G C}$, by $\mathrm{fc}(N, v, C)=\mathrm{f}\left(N, v, C, \underline{B_{0}}\right)$ for every $(N, v, C) \in \mathcal{G C}$. Since $\mathrm{f}$ satisfies $\mathrm{CE}$ and $\mathrm{FG}$, fc satisfies the two properties of the characterization of Myerson (1977). Then $\mathrm{fc}=\mathrm{SG}$, and $\mathrm{f}\left(N, v, C, \underline{B_{0}}\right)$ is uniquely determined. Note that we only use $\mathrm{CE}$ for graph-levels restricted games $(N, v, C, \underline{B})$ where $\underline{B}=\underline{B_{0}}$, i.e., where the levels structure is trivial.

Second, suppose that the payoffs according to $f$ are unique for any graph-levels restricted game with less than $k$ levels. Then, let $(N, v, C, \underline{B}) \in \mathcal{G C} \mathcal{L}$ be a graph-levels restricted game with $k$ levels, so that we have $\underline{B}=\left\{B_{0}, \ldots, B_{k+1}\right\}$. Let $i \in U \in B_{1}$. We show uniqueness of $\mathrm{f}_{i}(N, v, C, \underline{B})$ by a second induction on the cardinality of $U$.

On the one hand, suppose that $U=\{i\}$. Then, by CLG,

$$
\mathrm{f}_{i}(N, v, C, \underline{B})=\mathrm{f}_{U}\left(B_{1},\left(v^{C}\right)^{1}, C^{B_{1}}, \underline{B_{r}}\right) .
$$

Note that $\left(B_{1},\left(v^{C}\right)^{1}, C^{B_{1}}, \underline{B_{r}}\right)$ is a graph-levels restricted game with $k-1$ levels. Then, the payoff above is unique by the first induction hypothesis. On the other hand, suppose that the payoff according to $f$ is unique for any player that belongs to a union of the first level with a cardinality smaller than $u>1$, and let $i \in U \in B_{1} \in \underline{B}$ be such that $|U|=u$. Take $j \in U \backslash i$. By BC,

$$
\mathrm{f}_{i}(N, v, C, \underline{B})-\mathrm{f}_{j}(N, v, C, \underline{B})=\mathrm{f}_{i}\left(N, v, C, \underline{B^{-j}}\right)-\mathrm{f}_{j}\left(N, v, C, \underline{B^{-i}}\right) .
$$

Note that the payoffs in the right-hand side of the above equation are unique by the second induction hypothesis. Adding up Eq. (3) for every $j \in U \backslash i$, we obtain that

$$
u \cdot \mathrm{f}_{i}(N, v, C, \underline{B})-\sum_{j \in U} \mathrm{f}_{j}(N, v, C, \underline{B})
$$

is uniquely determined. Finally, by CLG,

$$
\sum_{j \in U} \mathrm{f}_{j}(N, v, C, \underline{B})=\mathrm{f}_{[U]}\left(B_{1},\left(v^{C}\right)^{1}, C^{B_{1}}, \underline{B_{r}}\right),
$$

which is also unique by the first induction hypothesis. Together with Eq. (4), this fact concludes this step of the proof.

\section{Logical independence}

It only remains to check that the four properties are independent. 
(i) Let $\mathrm{f}$ be the value on $\mathcal{G C L}$ defined for every $(N, v, C, \underline{B}) \in \mathcal{G C L}$ and $i \in N$ by $\mathrm{f}_{i}(N, v, C, \underline{B})=0$.

Then, $f$ satisfies $F G, B C$, and CLG but not $\mathrm{CE}$.

(ii) Let $\mathrm{f}$ be the value on $\mathcal{G C L}$ defined for every $(N, v, C, \underline{B}) \in \mathcal{G C} \mathcal{L}$ as follows: If $N=\{i, j\}$ and $C=C^{N}$, then

$$
\begin{aligned}
\mathrm{f}_{i}(N, v, C, \underline{B}) & =\frac{3}{4}(v(N)-v(j))+\frac{1}{4} v(i) \\
\mathrm{f}_{j}(N, v, C, \underline{B}) & =\frac{1}{4}(v(N)-v(i))+\frac{3}{4} v(j)
\end{aligned}
$$

Otherwise, $\mathrm{f}(N, v, C, \underline{B})=\mathrm{SG}^{\mathrm{L}}(N, v, C, \underline{B}) \cdot{ }^{10}$

Then, $f$ satisfies $\mathrm{CE}, \mathrm{BC}$, and CLG but not FG.

(iii) Let $\mathrm{f}$ be the value on $\mathcal{G C L}$ defined for every $(N, v, C, \underline{B}) \in \mathcal{G C L}$ by $\mathrm{f}(N, v, C, \underline{B})=$ $\mathrm{SG}^{\mathrm{L}}\left(N, v, C, \underline{B_{0}}\right)$.

Then, $f$ satisfies $\mathrm{CE}, \mathrm{FG}$, and $\mathrm{BC}$, but not CLG.

(iv) Let $\mathrm{f}$ be the value on $\mathcal{G C L}$ defined for every $(N, v, C, \underline{B}) \in \mathcal{G C L}$ and $i \in N$ by $\mathrm{f}_{i}(N, v, C, \underline{B})=\frac{\mathrm{SG}_{U}^{\mathrm{L}}\left(B_{1},\left(v^{C}\right)^{1}, C^{B_{1}}, \underline{B_{1}}\right)}{|U|}$, where $i \in U \in B_{1}$.

Then, $f$ satisfies $\mathrm{CE}$ (for $\underline{B_{0}}$ ), FG, and CLG, but not BC.

\section{Proof of Theorem 3.2.}

The proof proceeds in three steps. First, we prove that the Banzhaf levels graph value satisfies GI, FG, 2-E, NID, and 1-CLG. Second, we prove that there is at most one value on $\mathcal{G C L}$ satisfying GI, FG, 2-E, NID, and 1-CLG. Third, we prove that the five properties are logically independent.

\section{Existence}

First, we check that $\mathrm{BG}^{\mathrm{L}}$ satisfies GI. Let $(N, v, C, \underline{B}) \in \mathcal{G C} \mathcal{L}$ be such that $\{i\} \in$ $N / C$. Then, by definition of a game with graph-restricted communication, for every

\footnotetext{
${ }^{10}$ Here we assume that $i$ and $j$ can never be the label of any player that results from the merging of "core" players of the lowest level of the levels structure considered in CLG.
} 
$S \subseteq N \backslash i$, it holds that $v^{C}(S \cup i)-v^{C}(S)=v(i)$. Accordingly, $i$ is a dummy player in $\left(N, v^{C}\right)$ and $\mathrm{GI}$ then follows from the fact that $\mathrm{Ba}^{\mathrm{L}}$ satisfies the dummy player property (see pp 23-25 in Álvarez-Mozos and Tejada, 2011).

Second, we check that $\mathrm{BG}^{\mathrm{L}}$ satisfies 2-E. Let $(N, v, C, \underline{B}) \in \mathcal{G C} \mathcal{L}$ and $i, j \in U \in$ $B_{1} \in \underline{B}$ be such that $\{i: j\} \in C$. By definition of $P(i, \underline{B}), P(i, \underline{B}) \backslash\{j\}=P(j, \underline{B}) \backslash\{i\}$. Then, define $P(i j, \underline{B})=P(i, \underline{B}) \backslash\{j\}=\left\{T_{1}, \ldots, T_{m_{i j}}\right\}$ and $M_{i j}=\left\{1, \ldots, m_{i j}\right\}$, and note that $m_{i j}=m_{i}-1$. On the one hand,

$$
\begin{aligned}
& \mathrm{BG}_{i}^{\mathrm{L}}(N, v, C, \underline{B})+\mathrm{BG}_{j}^{\mathrm{L}}(N, v, C, \underline{B})=\mathrm{Ba}_{i}^{\mathrm{L}}\left(N, v^{C}, \underline{B}\right)+\mathrm{Ba}_{j}^{\mathrm{L}}\left(N, v^{C}, \underline{B}\right) \\
& =\sum_{R \subseteq M_{i j}} \frac{1}{2^{m_{i}}}\left[v^{C}\left(T_{R} \cup i\right)-v^{C}\left(T_{R}\right)+v^{C}\left(T_{R} \cup j \cup i\right)-v^{C}\left(T_{R} \cup j\right)\right] \\
& +\sum_{R \subseteq M_{i j}} \frac{1}{2^{m_{i}}}\left[v^{C}\left(T_{R} \cup j\right)-v^{C}\left(T_{R}\right)+v^{C}\left(T_{R} \cup i \cup j\right)-v^{C}\left(T_{R} \cup i\right)\right] \\
& =\sum_{R \subseteq M_{i j}} \frac{1}{2^{m_{i}-1}}\left[v^{C}\left(T_{R} \cup j \cup i\right)-v^{C}\left(T_{R}\right)\right] .
\end{aligned}
$$

On the other hand,

$$
\begin{aligned}
& \mathrm{BG}_{i}^{\mathrm{L}}\left(N, v_{i j}, C_{i j}, \underline{B}\right)=\mathrm{Ba}_{i}^{\mathrm{L}}\left(N,\left(v_{i j}\right)^{C_{i j}}, \underline{B}\right) \\
& =\sum_{R \subseteq M_{i}} \frac{1}{2^{m_{i}}}\left[\left(v_{i j}\right)^{C_{i j}}\left(T_{R} \cup i\right)-\left(v_{i j}\right)^{C_{i j}}\left(T_{R}\right)\right] \\
& =\sum_{R \subseteq M_{i j}} \frac{1}{2^{m_{i}}}\left[\left(v_{i j}\right)^{C_{i j}}\left(T_{R} \cup i\right)-\left(v_{i j}\right)^{C_{i j}}\left(T_{R}\right)+\left(v_{i j}\right)^{C_{i j}}\left(T_{R} \cup j \cup i\right)-\left(v_{i j}\right)^{C_{i j}}\left(T_{R} \cup j\right)\right] \\
& =\sum_{R \subseteq M_{i j}} \frac{1}{2^{m_{i}-1}}\left[v^{C}\left(T_{R} \cup j \cup i\right)-v^{C}\left(T_{R}\right)\right] .
\end{aligned}
$$


where the last equality follows from the following four observations:

$$
\begin{aligned}
& \left(v_{i j}\right)^{C_{i j}}\left(T_{R} \cup i\right)=\sum_{S \in\left(T_{R} \cup i\right) / C_{i j}} v_{i j}(S)=\sum_{S \in\left(T_{R} \cup i\right) / C_{i j}} v_{i j}(S \backslash j) \\
& =\sum_{S \in\left(T_{R} \cup i \cup j\right) / C} v_{i j}(S \backslash j)=\sum_{S \in\left(T_{R} \cup i \cup j\right) / C} v(S)=v^{C}\left(T_{R} \cup i \cup j\right) \text {, } \\
& \left(v_{i j}\right)^{C_{i j}}\left(T_{R}\right)=\sum_{S \in T_{R} / C} v_{i j}(S)=\sum_{S \in T_{R} / C} v(S)=v^{C}\left(T_{R}\right) \\
& \left(v_{i j}\right)^{C_{i j}}\left(T_{R} \cup i \cup j\right)=\sum_{S \in\left(T_{R} \cup i \cup j\right) / C_{i j}} v_{i j}(S)=v_{i j}(j)+\sum_{S \in\left(T_{R} \cup i \cup j\right) / C} v_{i j}(S \backslash j) \\
& =\sum_{S \in\left(T_{R} \cup i \cup j\right) / C} v(S)=v^{C}\left(T_{R} \cup i \cup j\right) \text {, } \\
& \left(v_{i j}\right)^{C_{i j}}\left(T_{R} \cup j\right)=\sum_{S \in\left(T_{R} \cup j\right) / C_{i j}} v_{i j}(S)=v_{i j}(j)+\sum_{S \in T_{R} / C} v_{i j}(S) \\
& =\sum_{S \in T_{R} / C} v(S)=v^{C}\left(T_{R}\right) \text {. }
\end{aligned}
$$

Taking into account that $j$ is isolated in $C_{i j}$, that $\mathrm{BG}^{\mathrm{L}}$ satisfies GI and that $v_{i j}(\{j\})=0$, it then follows that $\mathrm{BG}^{\mathrm{L}}$ satisfies 2-E.

Third, to verify that $\mathrm{BG}^{\mathrm{L}}$ satisfies $\mathrm{FG}$, we can replicate the argument in the proof of Theorem 3.1. Indeed, note that $\mathrm{Ba}^{\mathrm{L}}$ satisfies additivity and symmetry (see pp 24-25 in Álvarez-Mozos and Tejada, 2011).

Fourth, $\mathrm{BG}^{\mathrm{L}}$ satisfies NID by definition and by the fact that $\mathrm{Ba}^{\mathrm{L}}$ satisfies the level neutrality under individual desertion property (see pp 24-25 in Álvarez-Mozos and Tejada, 2011).

Fifth and last, to verify that $B G^{\mathrm{L}}$ satisfies $1-\mathrm{CLG}$, we can replicate the argument in the proof of Theorem 3.1, because Ba satisfies the singleton level game property (see pp 24-25 in Álvarez-Mozos and Tejada, 2011).

\section{Uniqueness}

Let $\mathrm{f}$ be a value on $\mathcal{G C} \mathcal{L}$ satisfying the five properties and $(N, v, C, \underline{B}) \in \mathcal{G C} \mathcal{L}$ be a graph-levels restricted game with $k$ levels. We show the uniqueness of $\mathrm{f}(N, v, C, \underline{B})$ by induction on $k$. 
First, let $k=0$, i.e., $\underline{B}=\underline{B_{0}}$. We show the uniqueness of $\mathrm{f}\left(N, v, C, \underline{B_{0}}\right)$ by induction on the number of links $|C|$. In the case $|C|=0$, every $i \in N$ is isolated in $C$, and hence $\mathrm{f}_{i}\left(N, v, C, \underline{B_{0}}\right)=v(i)$ follows from GI. Next, suppose that payoffs are unique for any graph-levels restricted game with a trivial levels structure and less than $c>0$ links, and let $\left(N, v, C, \underline{B_{0}}\right)$ be a graph-levels restricted game with $c$ links. Take $i \in N$. If $i$ is isolated in the graph, uniqueness follows again from GI. Otherwise, i.e. if $i$ is not isolated in the graph, let $j \in N$ be such that $\{i: j\} \in C$. On the one hand, by FG

$$
\mathrm{f}_{i}\left(N, v, C, \underline{B_{0}}\right)-\mathrm{f}_{j}\left(N, v, C, \underline{B_{0}}\right)=\mathrm{f}_{i}\left(N, v, C^{-i j}, \underline{B_{0}}\right)-\mathrm{f}_{j}\left(N, v, C^{-i j}, \underline{B_{0}}\right) .
$$

On the other hand, by 2-E

$$
\mathrm{f}_{i}\left(N, v, C, \underline{B_{0}}\right)+\mathrm{f}_{j}\left(N, v, C, \underline{B_{0}}\right)=\mathrm{f}_{i}\left(N, v, C_{i j}, \underline{B_{0}}\right)+\mathrm{f}_{j}\left(N, v, C_{i j}, \underline{B_{0}}\right) .
$$

By definition, both modified communication graphs above, namely $C^{-i j}$ and $C_{i j}$, have less than $c$ links. Then, the payoffs in the right-hand side of Eqs. (5) and (6) are uniquely determined by the second induction hypothesis. Consequently, the payoffs in the lefthand side are also determined, thereby implying the uniqueness of $\mathrm{f}_{i}\left(N, v, C, \underline{B_{0}}\right)$ and $\mathrm{f}_{j}\left(N, v, C, \underline{B_{0}}\right)$-and hence that of $\mathrm{f}\left(N, v, C, \underline{B_{0}}\right)$. Note that uniqueness follows because Eqs. (5) and (6) define a determinate compatible system of equations in the two unknowns $\mathrm{f}_{i}\left(N, v, C, \underline{B_{0}}\right)$ and $\mathrm{f}_{j}\left(N, v, C, \underline{B_{0}}\right)$.

Second, suppose that the payoffs according to $f$ are unique for any graph-levels restricted game with less than $k>0$ levels. Let $(N, v, C, \underline{B}) \in \mathcal{G C L}$ be a graph-levels restricted game with $k$ levels, i.e., $\underline{B}=\left\{B_{0}, \ldots, B_{k+1}\right\}$. Take $i \in U \in B_{1}$. We show the uniqueness of $\mathrm{f}_{i}(N, v, C, \underline{B})$ by a second induction on the cardinality of $U$. On the one hand, suppose that $U=\{i\}$. Then, by 1-CLG

$$
\mathrm{f}_{i}(N, v, C, \underline{B})=\mathrm{f}_{[U]}\left(\left[B_{1}\right],\left(v^{C}\right)^{1}, C^{\left[B_{1}\right]}, \underline{B_{r}}\right) .
$$

Note that $\left(\left[B_{1}\right],\left(v^{C}\right)^{1}, C^{\left[B_{1}\right]}, \underline{B_{r}}\right)$ is a graph-levels restricted game with $k-1$ levels. Then, the payoff above is unique by the first induction hypothesis. On the other hand, suppose that the payoff according to $f$ is unique for any player that belongs to a union 
of the first level with a cardinality smaller than $u>1$ and let $i \in U \in B_{1}$ be such that $|U|=u$. Take $j \in U \backslash i$. Then, by NID

$$
\mathrm{f}_{i}(N, v, C, \underline{B})=\mathrm{f}_{i}\left(N, v, C, \underline{B^{-j}}\right) .
$$

The payoff $\mathrm{f}_{i}\left(N, v, C, \underline{B^{-j}}\right)$ is unique by the second induction hypothesis, which concludes this step of the proof.

\section{Logical independence}

It only remains to check that the five properties are independent.

(i) Let $\mathrm{f}$ be the value on $\mathcal{G C L}$ defined for every $(N, v, C, \underline{B}) \in \mathcal{G C} \mathcal{L}$ and $i \in N$ by $\mathrm{f}_{i}(N, v, C, \underline{B})=0$.

Then, f satisfies FG, 2-E, NID, and 1-CLG but not GI.

(ii) Let $\mathrm{f}$ be the value on $\mathcal{G C} \mathcal{L}$ defined for every $(N, v, C, \underline{B}) \in \mathcal{G C} \mathcal{L}$ as follows: If $N=\{i, j\}$ and $C=C^{N}$, then

$$
\begin{aligned}
\mathrm{f}_{i}(N, v, C, \underline{B}) & =\frac{3}{4}(v(N)-v(j))+\frac{1}{4} v(i) \\
\mathrm{f}_{j}(N, v, C, \underline{B}) & =\frac{1}{4}(v(N)-v(i))+\frac{3}{4} v(j)
\end{aligned}
$$

Otherwise, $\mathrm{f}(N, v, C, \underline{B})=\mathrm{BG}^{\mathrm{L}}(N, v, C, \underline{B})$.

Then, f satisfies GI, 2-E, NID, and 1-CLG ${ }^{11}$ but not FG.

(iii) Let $\mathrm{f}$ be the value on $\mathcal{G C} \mathcal{L}$ defined for every $(N, v, C, \underline{B}) \in \mathcal{G C} \mathcal{L}$ and $i \in N$ by $\mathrm{f}_{i}(N, v, C, \underline{B})=\sum_{R \subseteq M_{i}} \frac{|R| !\left(m_{i}-|R|-1\right) !}{m_{i} !}\left[v^{C}\left(T_{R} \cup i\right)-v^{C}\left(T_{R}\right)\right]$.

Then, $f$ satisfies GI, FG, NID and 1-CLG, but not 2-E.

(iv) Let $\mathrm{f}$ be the value on $\mathcal{G C} \mathcal{L}$ defined for every $(N, v, C, \underline{B}) \in \mathcal{G C} \mathcal{L}$ by $\mathrm{f}(N, v, C, \underline{B})=$ $\operatorname{BG}^{\mathrm{L}}\left(N, v, C, \underline{B_{0}}\right)$.

Then, f satisfies GI, FG, 2-E, and NID but not 1-CLG.

\footnotetext{
${ }^{11}$ The same remark as in Footnote 10 applies here.
} 
(v) Let $\mathrm{f}$ be the value on $\mathcal{G C L}$ defined for every $(N, v, C, \underline{B}) \in \mathcal{G C} \mathcal{L}$ and $i \in N$ by $\mathrm{f}_{i}(N, v, C, \underline{B})=\frac{\mathrm{BG}_{U}^{\mathrm{L}}\left(B_{1},\left(v^{C}\right)^{1}, C^{B_{1}}, \underline{B_{1}}\right)}{|U|}$, where $i \in U \in B_{1}$.

Then, $\mathrm{f}$ satisfies GI (for $\underline{B_{0}}$ ), FG, 2-E, and 1-CLG but not NID. 